\title{
Adolescent girls and young women's perceptions of oral pre- exposure prophylaxis introduction in Tanzania: Findings from implementation science research
}

Population Council

Follow this and additional works at: https://knowledgecommons.popcouncil.org/departments_sbsr-hiv

Part of the Demography, Population, and Ecology Commons, Family, Life Course, and Society Commons, International Public Health Commons, Medicine and Health Commons, and the Women's Health Commons How does access to this work benefit you? Let us know!

\section{Recommended Citation}

Population Council. 2017. "Adolescent girls and young women's perceptions of oral pre-exposure prophylaxis introduction in Tanzania: Findings from implementation science research," DREAMS Tanzania Results Brief 3. Washington, DC: Population Council. 


\title{
ADOLESCENT GIRLS AND YOUNG WOMEN'S PERCEPTIONS OF ORAL PRE-EXPOSURE PROPHYLAXIS INTRODUCTION IN TANZANIA
}

\author{
FINDINGS FROM IMPLEMENTATION SCIENCE RESEARCH
}

The World Health Organization recommends that oral pre-exposure prophylaxis (PrEP), antiretroviral medication taken by HIV-uninfected persons to prevent HIV, be offered as a prevention choice for people at substantial risk of HIV infection. Adolescent girls and young women (AGYW) in Tanzania are particularly vulnerable to HIV infection due to several factors including early marriage, having older sexual partners, and challenges in negotiating safe sex. ${ }^{1}$ HIV prevalence among adolescent girls aged $15-19$ years is 1.3 percent, and nearly four times higher (4.4 percent) among young women aged $20-24 .{ }^{1}$ PrEP has the potential to substantially reduce HIV acquisition among AGYW in Tanzania as part of a comprehensive HIV prevention package, provided they can access, use, and adhere to the medication. ${ }^{2-5}$

Policymakers, programmers, and other stakeholders are currently considering PrEP for AGYW in Tanzania. Understanding AGYW's attitudes and perceptions about PrEP is critical to ensuring that introduction is done is a manner that reduces barriers and optimizes facilitators of PrEP acceptance and use.

The Population Council, in collaboration with the National AIDS Control Programme (NACP) and CSK Research Solutions Ltd, is conducting implementation science research in Tanzania to identify key considerations for introducing PrEP to AGYW. The research sites consist of one urban and one rural setting in Dar Es Salaam and Mbeya districts, respectively. This brief presents results from our qualitative in-depth interviews (IDIs) and focus group discussions (FGDs) with AGYW.

\section{METHODS}

Study team researchers conducted 24 IDIs and 4 FGDs in Kiswahili with sexually active, HIV-negative AGYW aged 15-24 years. FGDs consisted of unmarried AGYW while IDIs included married AGYW and unmarried female sex workers (FSWs). Trained research assistants used a standardized script to provide detailed information on PrEP to all AGYW. Placebo PrEP pills were shown to AGYW as part of the interviews. This study was approved by the Population Council Institutional Review Board (New York, USA) and the National Institute of Medical Research (Dar Es Salaam, Tanzania).

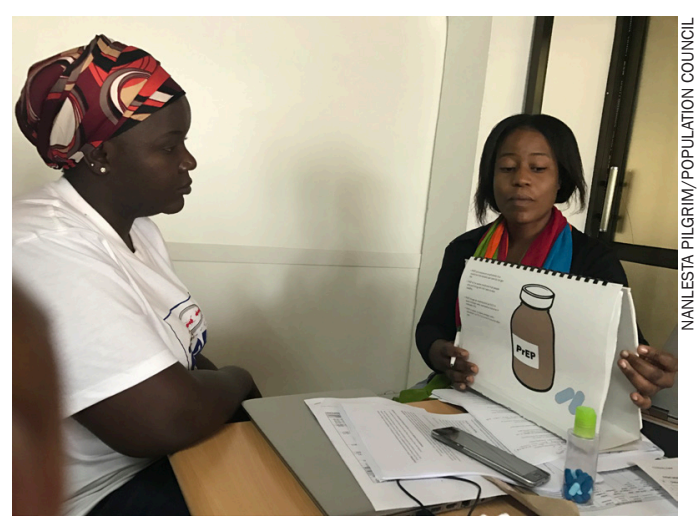

To effectively introduce PrEP to AGYW, we need to understand their lived experiences and engage them in research, program planning, and policy making around PrEP.

\section{KEY FINDINGS AND RECOMMENDATIONS}

Rollout PrEP to AGYW: AGYW express a strong need for PrEP as they perceive themselves at high risk of HIV because of lack of trust in relationships and unequal gender power dynamics.

Address barriers to use: These include AGYW's fear of stigma, side effects, and the impact of PrEP on their relationship.

\section{Enact and promote facilitators of}

adherence: Community education and sensitization about PrEP, knowing one is HIV negative from routine testing, being able to access PrEP free or at low cost, and integrating PrEP within other health services would facilitate PrEP adherence by AGYW. 


\begin{tabular}{|c|c|c|}
\hline \multicolumn{2}{|l|}{ IDI participants } & FGD participants \\
\hline 24 & Number of AGYW & 36 \\
\hline $19-24$ years & Age range of participants & $15-24$ years \\
\hline $\begin{array}{l}12 \text { married/living with } \\
\text { partner } \\
12 \text { never married }\end{array}$ & Marital status & 36 never married \\
\hline $\begin{array}{l}15 \text { primary school } \\
9 \text { secondary school }\end{array}$ & $\begin{array}{l}\text { Highest education } \\
\text { level attained }\end{array}$ & $\begin{array}{l}24 \text { primary school } \\
12 \text { secondary school }\end{array}$ \\
\hline $\begin{array}{l}12 \text { sex work } \\
5 \text { business } \\
5 \text { domestic } \\
1 \text { farming } \\
1 \text { unemployed }\end{array}$ & Occupation & $\begin{array}{r}28 \text { domestic } \\
6 \text { business } \\
2 \text { farming }\end{array}$ \\
\hline
\end{tabular}

\section{RESULTS}

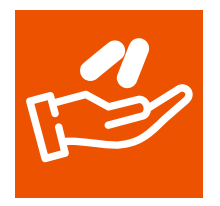

\section{AGYW express a strong need for PrEP as they perceive themselves at high risk of HIV}

Study participants have a sophisticated awareness of their own risk and their sexual partner's risk of HIV, but often feel powerless to change their own behavior.

- AGYW, regardless of partnership status, express a high level of anxiety about becoming infected with HIV due to distrust surrounding their partner's sexual behaviors outside of their relationship. There are strong cultural and gender norms that prohibit a woman from refusing to have sex with her husband or a long-term partner.

\section{4 ...I am aware that at any moment I could get the HIV infection. ...I am not sure of where he has been..., he might have contracted the disease from there. Since he is my partner and I depend on him, I fail to resist. But frankly speaking, my thoughts are usually on being HIV infected during such occasions.}

-FSW, IDI, age 23, Dar es Salaam

\section{Things that will put me at risk for HIV are such as my own partner, because I will not be sure of what he is doing out there, he might be hanging out with other women.}

He is my husband he will need to have sex with me, I cannot resist him, I must accept his requests.

-Married AGYW, IDI, age 19, Mbeya

- FSWs, in particular, view themselves at high risk of HIV due to such factors as lack of condom use with clients, high reported instances of unprotected anal sex, sharing clients among one another, and sexual violence.

\begin{abstract}
46 Men have different behaviors. You may agree upon doing some things before having sex but when you get into a private room, some of the men breach the agreement and do whatever they wish. He can force you to have anal sex. We do face a lot of challenges.
\end{abstract}

-FSW, IDI, age 23, Mbeya

- All AGYW report that PrEP would alleviate their daily anxiety about becoming infected with HIV as they would be able to control their risk of HIV.

$$
\begin{aligned}
& \text { Using PrEP is easier because it is just } \\
& \text { about myself. I can decide to use PrEP } \\
& \text { unlike condoms where you may want to use } \\
& \text { but your partner doesn't. Using PrEP is easier } \\
& \text { than condoms. } \\
& \text {-Married AGYW, IDI, age 20, Mbeya }
\end{aligned}
$$




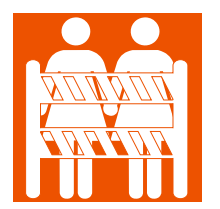

Fear of stigma, side effects, and the impact of PrEP on their relationship are the key barriers noted by AGYW

- Fear of HIV stigma from peers, parents, and sexual partner(s) is a commonly cited barrier to taking PrEP. Many fear that PrEP may be mistaken for ARVs leading to the misconception that they are HIV infected. Two participants commented:

$$
4
$$
If you take these pills, your friends will isolate you knowing that you are HIV infected. Even men won't seduce you anymore. Same to women, they won't accept a man who is taking these pills.

\section{They will think differently because PrEP pills are similar to HIV pills, so people will think you are using ARVs.}

-FGD, ages 15-17, Dar es Salaam

- Some AGYW note that their PrEP use could lead to relationship dissolution, loss of financial support, and violence from their male partners. Respondents note that if PrEP was used covertly without the partner's knowledge, it would raise issues around trust and fidelity.

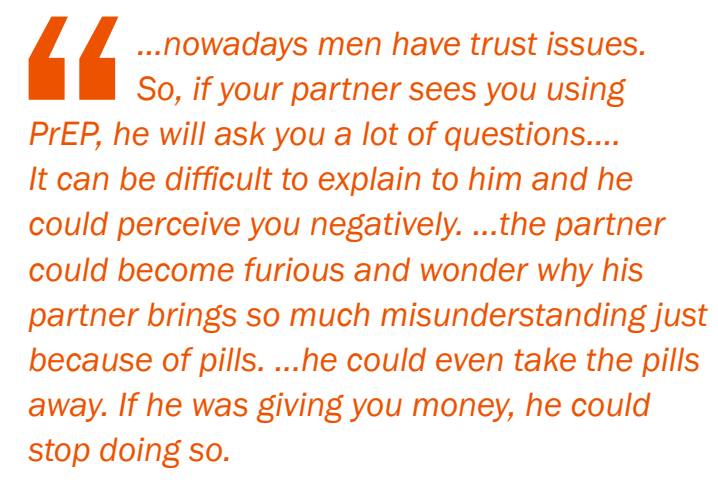

-FSW, IDI, age 19, Dar es Salaam

- Potential side effects of PrEP are also a key concern for many AGYW, who feel that they would negatively impact their ability to adhere to the daily regimen. Additionally, AGYW express concerns about PrEP's potential impact on their ability for women to bear children in the future, which is especially concerning to unmarried AGYW and married AGYW without children. these medications do cause a lot of effects especially for us who have not yet given birth. They can be worried about reproductive ability thereafter in the future.

-FSW, IDI, age 23, Dar es Salaam

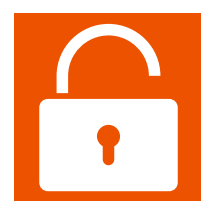

Key facilitators to AGYW PrEP adherence are community education and sensitization, knowing one's HIV status, and availability of PrEP free of charge and in conjunction with other services

- Male partner, parental, and community support and sensitization around the uses and benefits of PreP would encourage AGYW's use of PrEP. If these key constituents are properly educated about the use for PrEP, AGYW feel that they would encourage and support their continued use of PrEP.

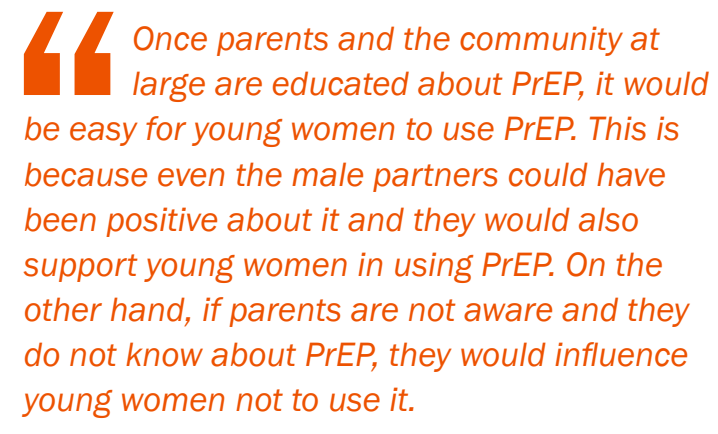

-FGD, ages 18-24, Dar es Salaam

- AGYW report that the relief associated with knowing they are HIV negative due to the 3-month HIV testing requirement associated with taking PrEP would encourage them to continue adhering to PrEP.

\section{4 ...if you use this medication, you will test negative for HIV when you go for test, you protect yourself against HIV. You know they don't want to be infected with HIV. If you tell them in such a way, they accept it quickly. \\ -Married AGYW, IDI, age 21, Dar es Salaam}

- AGYW prefer that PrEP be offered in comprehensive or multiple settings, such as public and private hospitals, dispensaries, and pharmacies, as these venues would make it less apparent which specific services they were seeking. Moreover, these settings offer both 
care through knowledgeable health staff, including availability of female staff, counseling on proper use of medicines, HIV testing and counseling, as well as other services such as family planning.

\section{4} At hospital is where medicines are being provided, there is counselling services, and there are many doctors. Also, it is the entry point for many service providers. Visiting the health center will be accompanied by seeing a doctor who will provide counseling to us and we will learn and understand many things.

$$
\text { -FSW, IDI, age 21, Mbeya }
$$

\section{- Most AGYW report they would take PrEP if it were} offered free of charge. High costs would deter consistent, long term use of PrEP. Some AGYW are willing to pay a maximum of 200TSH (\$.09USD) per pill to $10,000 T S H(\$ 4.47$ USD) for a 1-month supply of PrEP. Only a few participants say they could manage to find a way to use PrEP regardless of the cost. In response to a question if cost would cause the respondent to fail to use PrEP:

41 Yes, I will end up having unprotected sex. ...it will depend on the financial position of a person. PrEP can be available at the health facility at cost. Whoever is able to pay will buy it. Those who are not able to pay will die.

\section{RECOMMENDATIONS}

- Ensure that demand creation activities include sensitization and education of communities, parents, and male partners of AGYW.

- Train providers to deliver adequate education about PrEP to avoid misperceptions about its intended use, side effects, and efficacy.

- Increase access to PrEP by integrating it into existing comprehensive health services, such as hospitals, dispensaries, and pharmacies, at no or low cost to ensure widespread coverage and uptake by the most vulnerable populations.

\section{REFERENCES}

${ }^{1}$ Tanzania Commission for AIDS (TACAIDS) et al. 2013. Tanzania HIV/ AIDS and Malaria Indicator Survey 2011-12. Dar es Salaam, Tanzania: TACAIDS, ZAC, NBS, OCGS, and ICF International.

${ }^{2}$ WHO. 2015. "Guideline on when to start antiretroviral therapy and on pre-exposure prophylaxis for HIV." Geneva, Switzerland: WHO.

${ }^{3}$ Fonner, V. A. et al. 2016. "Effectiveness and safety of oral HIV preexposure prophylaxis for all populations," AIDS 30(12): 1973.

${ }^{4}$ Van Damme, L. et al. 2012. "Preexposure prophylaxis for HIV infection among African women," New England Journal of Medicine 367(5): 411-422.

${ }^{5}$ Corneli, A. L. et al. 2014. “FEM-PrEP: adherence patterns and factors associated with adherence to a daily oral study product for pre-exposure prophylaxis," Journal of Acquired Immune Deficiency Syndromes 66(3): 324-331.
For more information, contact Nanlesta Pilgrim (npilgrim@ popcouncil.org), Lou Apicella (lapicella@popcouncil.org), or Catherine Kahabuka (ckahabuka@cskresearch.com).
POPULATION COUNCIL

Ideas. Evidence. Impact.
The Population Council confronts critical health and development issues-from stopping the spread of HIV to improving reproductive health and ensuring that young people lead full and productive lives. Through biomedical, social science and public health research in about 50 countries, the Council works with our partners to deliver solutions that lead to more effective policies, programs, and technologies to improve lives worldwide. Established in 1952 and headquartered in New York, the Council is a nongovernmental, nonprofit organization with an international board of trustees. popcouncil.org

Suggested citation: Population Council. 2017. "Adolescent girls and young women's perceptions of oral pre-exposure prophylaxis introduction in Tanzania: findings from implementation science research," DREAMS Tanzania Results Brief 3. Washington, DC: Population Council. 